\title{
THE USE OF DIFFERENTIAL COEFFICIENTS IN THE DEVELOPMENT AND INTERPRETATION OF QUANTITATIVE GENETIC MODELS*
}

\author{
A. J. WRIGHT \\ Plant Breeding Institute, Cambridge
}

Received 8.ii.79

\begin{abstract}
Summary
The polynomial regression of genotypic value on to allele frequency for a biallelic locus of any ploidy in a randomly mating population is shown to be equivalent to a Taylor series, and the components of genetic variance are expressed as simple functions of the various differential coefficients of the population mean. A straightforward extension allows a similar formulation of epistatic variances. The simplicity of this method of deriving expressions for all genetic variances is illustrated by reference to an autotetraploid model, and for diploids with epistasis.

Other uses of these differential coefficients include the prediction of patterns of selection response and the development of prediction formulae for the mean values of composite populations.
\end{abstract}

\section{Introduction}

THE general method of subdivision of the genetic variance in randomly mating populations developed by Kempthorne (1957) and others depends on the fitting of the effects of single alleles and their successively higher orders of interaction by least squares. For the case of an autotetraploid locus with two alleles, $\mathrm{Li}$ (1957) proposed a different method by which the components are found as the variance associated with successive terms in a polynomial regression of genotype value on to allele frequency.

Although less comprehensive in their representation of real populations, two allele models do have the advantage that they can be expressed in terms of relatively few parameters which deal with genetic phenomena at the level of the gene rather than the population, thus allowing more fundamental analysis. However, these genic models typically give rise to complex expressions for genetic variances in randomly mating populations, especially when epistasis is considered (Mather, 1974).

The purpose of this paper is to show that the formulation of genetic variances with respect to any chosen two-allele model is considerably simplified by the use of differential calculus. In addition, the differential coefficients have an interpretative value of their own, and can lead to predictions about the behaviour of populations under selection and when intercrossed to form composites.

* Presented in part at the Third meeting of the Eucarpia Biometrics section, 1978. 43/1-A 


\section{The DERIVATION OF Genetic VARIANCES}

(i) Single locus models

Li (1957) showed how the components of variance associated with the effects of alleles and their three orders of interaction at a single biallelic tetraploid locus in a randomly mating population correspond to the variances associated with successive terms in a polynomial regression of genotype on to gene frequency. A novel method of differencing of genotypic values was devised which allowed a formulation of the covariance associated with each term, and the regression variance (sum of squares) was arrived at conventionally by way of the regression coefficient.

In general, the genotypic value of any individual with respect to a single locus can be expanded as a polynomial of order $n$, where $n$ is the level of ploidy. In this regression, each successive term is fitted independently of those preceding, so that the independent variates are not $x, x^{2}, \ldots$, but the orthogonal functions $\xi_{1}=\alpha+x, \quad \xi_{2}=\alpha_{2}+\beta x+x^{2}$ et cetera (Dalebroux, 1962). Furthermore, the relationship being expressed is not approximate, or empirical, but is a completely deterministic model where genotype is an exact function of gene frequency. It can therefore be regarded as equivalent to a Taylor series, where each successive term modifies the existing equation to increase its accuracy of prediction. Hence

$$
y=\bar{y}+(d y / d x)\left(x_{i}-\bar{x}\right)+\left(d^{2} y / d x^{2}\right)\left(x_{i}-\bar{x}\right)^{2} / 2 !+\ldots+\left(d^{n} y / d x^{n}\right)\left(x_{i}-\bar{x}\right)^{n} / n !
$$

where $(d y / d x)$ terms are differential coefficients evaluated at $\bar{x}$. The relationship between these differential coefficients and genetic effects and their interactions in diploids has been recognised by Arunachalam and Owen (1971). The analogy between the regression model and Taylor series might be expected to lead to an even simpler method of formulation of genetic variances than that used by $\mathrm{Li}$, because the $r$ th regression coefficient can now be found directly as $\left(d^{r} y / d x^{r}\right) / r$ !

The necessary equivalence between regression and Taylor series and their associated sums of squares can be established in terms of the present genetic problem as follows. Using the condensed notation given by Arunachalam and Owen (1971), the mean of the biallelic population of ploidy $n$ is given by expansion of $(p A+q a)^{n}$, where the value of any genotype is represented by its genetic constitution (e.g. $A a)$ and $p$ and $q$ are the frequencies of the two alleles $A$ and $a(p+q=1)$. Considering the identity

$$
\left(p A^{2}+q a^{2}\right)^{n}=\left((p A+q a)^{2}+p q(A-a)^{2}\right)^{n},
$$

expansion of the left-hand side gives the total sum of squares of the population of genotypes. The first term arising on expansion of the right-hand side is $(p A+q a)^{2 n}$, which is the square of the population mean (or correction term), so that the remainder must represent an orthogonal partitioning of the population sum of squares. These following terms are all of the form

$$
\left(\begin{array}{l}
n \\
r
\end{array}\right) p^{r} q^{r}(p A+q a)^{2(n-r)}(A-a)^{2 r} \text {, where } 1 \leqq r \leqq n .
$$

Since the $r$ th derivative of the population mean is readily found to be

$$
n(n-1)(n-2) \ldots(n-r+1)(p A+q a)^{n-r}(A-a)^{r},
$$


the sum of squares (or variance) associated with the $r$ th term in the polynomial is

$$
p^{r} q^{r}\left(d^{r} y / d p^{r}\right)^{2} /\left(\begin{array}{l}
n \\
r
\end{array}\right) r !^{2}
$$

This final form provides an extremely simple and convenient method of formulation of genetic variances for any level of ploidy, as the problem is essentially reduced to that of writing the mean of the randomly mating population in terms of any chosen model and carrying out repeated differentiation with respect to $p$.

\section{(ii) Multiple locus models}

The same approach can easily be extended to cater for epistasis by reference to a multiple Taylor series where $y$ is a function of allelic frequencies $p$ and $u(v=1-u)$ at two loci $(A$ and $B)$ in linkage equilibrium. Using the identity:

$$
\begin{aligned}
\left(p A^{2}+q a^{2}\right)^{n}\left(u B^{2}+\right. & \left.v b^{2}\right)^{n} \\
& =\left((p A+q a)^{2}+p q(A-a)^{2}\right)^{n}\left((u B+v b)^{2}+u v(B-b)^{2}\right)^{n}
\end{aligned}
$$

the resulting variances are of the form

$$
\left(\begin{array}{l}
n \\
r
\end{array}\right)\left(\begin{array}{l}
n \\
s
\end{array}\right) p^{r} q^{r} u^{s} v^{s}(p A+q a)^{2(n-r)}(A-a)^{2 r}(u B+v b)^{2(n-s)}(B-b)^{2 s}
$$

Those involving single locus effects can now be written as before, but the new variance for epistatic interaction between allelic interactions of the $r$ th order at $A$ and the $s$ th at $B$ becomes

$$
p^{r} q^{r} u^{s} v^{s}\left(\partial^{m} y / \partial p^{r} . \partial u^{s}\right)^{2} /\left(\begin{array}{l}
n \\
r
\end{array}\right)\left(\begin{array}{l}
n \\
s
\end{array}\right)(r ! s !)^{2}
$$

where $(\partial y / \partial p)$ is a partial derivative and $m=r+s$.

\section{(iii) Examples}

The simplicity of this method can best be shown by reference to examples, and for this purpose the cases of autotetraploids without epistasis and diploids with epistasis have been chosen.

\section{(a) Autotetraploids}

The model chosen for parametrisation of gene effects is that used by Easton (1976), as shown in table 1. The mean of the randomly bred

TABLE 1

The model used for parametrisation of gene effects with tetraploid inheritance (Easton, 1976)

\begin{tabular}{lccccc}
\multicolumn{5}{c}{ Genotypes } \\
& $A^{4}$ & $A^{3} a$ & $A^{2} a^{2}$ & $A a^{3}$ & $a^{4}$ \\
$h$ & 2 & 1 & 0 & -1 & -2 \\
$v$ & 0 & 3 & 4 & 3 & 0 \\
$w$ & 0 & 1 & 0 & -1 & 0 \\
& 0 & 1 & 0 & 1 & 0
\end{tabular}


population in terms of a single gene is a polynomial of the fourth order:

$$
y=2(p-q) d+12 p q h+4 p q(p-q) v+4 p q\left(p^{2}+q^{2}\right) w .
$$

Repeated differentiation with respect to $p$ (remembering that $q=1-p$ ) leads to successive variance components according to (l) with $r$ taking successive values of 1 to 4 :

$$
\begin{aligned}
& V_{A}=p q\left(4\left(d+3(q-p) h+(6 p q-1) v+(q-p)^{3} w\right)\right)^{2} /(4 \times 1 \times 1) \\
& =4 p q\left(d+3(q-p) h+(6 p q-1) v+(q-p)^{3} w\right)^{2}, \\
& V_{D}=p^{2} q^{2}\left(-24\left(h-(q-p) v+(q-p)^{2} w\right)\right)^{2} /(6 \times 2 \times 2) \\
& =24 p^{2} q^{2}\left(h-(q-p) v+(q-p)^{2} w\right)^{2} \\
& V_{T}=p^{3} q^{3}(48(v+2(p-q) w))^{2} /(4 \times 6 \times 6)=16 p^{3} q^{3}(v+2(p-q) w)^{2} \\
& V_{Q}=p^{4} q^{4}(-192 w)^{2} /(1 \times 24 \times 24)=64 p^{4} q^{4} w^{2}
\end{aligned}
$$

These expectations are identical to those given by Easton (1976) or as found using the method given by Li. In the absence of epistasis, the total variance for each component is given simply by summation over loci.

\section{(b) Diploids with epistasis}

In this case a diploid model is assumed in which loci interact in pairs. Eight parameters are used by Mather and Jinks (1971) to describe the effects of a pair of interacting loci with allele frequencies of $p$ and $q$ at the first and $u$ and $v$ at the second. In addition to $d$ and $h$ terms used to represent the effects of homozygosity and heterozygosity at each locus, $i$ indicates the interaction of homozygous states at both loci, $l$ that of heterozygous states, and $j_{a}$ and $j_{b}$ homozygous $\times$ heterozygous interactions where the suffix refers to the locus ( $a$ or $b$ ) which is homozygous.

The population mean can thus be written as a second-order polynomial in $p$ and $u$ :

$$
\begin{aligned}
y=(p-q) d_{a}+2 p q h_{a}+(u-v) d_{b} & +2 u v h_{b}+(p-q)(u-v) i \\
& +2 p q(u-v) j_{b}+2 u v(p-q) j_{a}+4 p q u v l .
\end{aligned}
$$

Differentiation with respect to $p$ and application of (1) gives:

$$
V_{A_{a}}=2 p q\left(d_{a}+(u-v) i+2 u v j_{a}+(q-p)\left(h_{a}+(u-v) j_{b}+2 u v l\right)\right)^{2},
$$

and then

$$
V_{D_{a}}=4 p^{2} q^{2}\left(h_{a}+(u-v) j_{b}+2 u v l\right)^{2},
$$

while similar differentiation with respect to $u$ leads to:

and

$$
V_{A_{b}}=2 u v\left(d_{b}+(p-q) i+2 p q j_{b}+(v-u)\left(h_{b}+(p-q) j_{a}+2 p q l\right)\right)^{2},
$$

$$
V_{D_{b}}=4 u^{2} v^{2}\left(h_{b}+(p-q) j_{a}+2 p q l\right)^{2} .
$$

The mixed derivatives $\partial y^{2} / \partial p \partial u, \partial y^{3} / \partial p^{2} . \partial u, \partial y^{3} / \partial p . \partial u^{2}$, and $\partial y^{4} / \partial p^{2} \cdot \partial u^{2}$, after appropriate application of (2), lead to the components of epistatic variation:

$$
V_{A A}=4 p q u v\left(i+(q-p) j_{b}+(v-u) j_{a}+(q-p)(v-u) l\right)^{2},
$$


and

$$
\begin{aligned}
& V_{A D}=8 p q u^{2} v^{2}\left(j_{a}+(q-p) l\right)^{2}, \\
& V_{D A}=8 p^{2} q^{2} u v\left(j_{b}+(v-u) l\right)^{2},
\end{aligned}
$$

$$
V_{D D}=16 p^{2} q^{2} u^{2} v^{2} l^{2}
$$

The accumulated effects of all genes segregating are accounted for by summation over all genes for variances involving single genes (i.e. $V_{A_{a}}$, $V_{A_{b}}, V_{D_{a}}$, and $V_{D_{b}}$ ), and over all interacting pairs for epistatic components. This procedure leads to expectations identical to those given by Mather (1974). No change in principle is involved if parameters are specified to allow for interactions of loci in sets of three or more. As in the case of tetraploidy, these components of variation are associated with the average effects and various types of interaction of alleles, and are identical with those defined by Kempthorne (1957).

\section{OTHER USES OF THE DIFFERENTIAL GOEFFICIENTS}

Apart from their use in providing a simple and reliable formulation of genetic variances, the differential coefficients have an intrinsic interest of their own, as they specify how a randomly mating population responds to changes in allele frequency. This is clearly of relevance in selection, but can also be used in comparing the expected means of populations with different allele frequencies, as is the case with composite varieties.

\section{(i) Selection}

\section{(a) Patterns of response}

The importance of additive genetic variance in the prediction of shortterm selection response is well known. This is due to its close association with the first differential which relates changes of gene frequency to changes in population mean. With respect to a single locus with two alleles, response to selection involves two processes; a change in gene frequency following selection, and a consequent change in population mean. These two components have the values $\frac{1}{2} p q\left(\frac{d y}{d p}\right) / \sigma_{x}^{2}$ and $(d y / d p)$ (where $x$ is the criterion of selection), and their product $2 p q \alpha^{2} / \sigma_{x}^{2}$ is the familiar narrow sense heritability coefficient (Falconer 1960).

Since they depend only on the first-order derivative, such predictions of selection response are strictly valid only over a small range of gene frequency changes where non-linearity can be ignored. Longer-term predictions would require a consideration of how the relationship between gene frequency and population mean itself changes, and hence of the higher order derivatives.

As examples, table 2 gives the expectations of the various differential coefficients for a pair of diploid loci exhibiting classical complementary and duplicate epistasis. These are derived from the formulae in Section (2) by putting $d_{a}=d_{b}=h_{a}=h_{b}=i=j_{a}=j_{b}=l=1$ for complementary interaction and by changing the sign of $i, j_{a}, j_{b}$ and $l$ for duplicate epistasis (Mather and Jinks, 1971). 
TABLE 2

Differential coefficients with duplicate and complementary types of epistasis

$\begin{array}{lcc}(d y / d p) & 8 q v^{2} & 8 q\left(1-v^{2}\right) \\ (d y / d u) & 8 q^{2} v & 8\left(1-q^{2}\right) v \\ \left(d^{2} y / d p^{2}\right) & -8 v^{2} & -8\left(1-v^{2}\right) \\ \left(d^{2} y / d u^{2}\right) & -8 q^{2} & -8\left(1-q^{2}\right) \\ \left(d^{2} y / d p d u\right) & -16 q v & 16 q v \\ \left(d^{3} y / d p^{2} d u\right) & 16 v & -16 v \\ \left(d^{3} y / d p d u^{2}\right) & 16 q & -16 q \\ \left(d^{4} y / d p^{2} d u^{2}\right) & -16 & 16\end{array}$

For both types of epistasis, the second-order differentials $d^{2} y / d p^{2}$ and $d^{2} y / d u^{2}$ are both negative, as they would be for a model without epistasis. This represents the well known fact that the response due to a dominant allele is progressively reduced as it approaches fixation. The two models do differ markedly with respect to the mixed derivative $d^{2} y / d p . d u$, however, as this is positive in the case of complementary interaction, but negative for duplicate epistasis. Hence it can be expected that advances in gene frequency at one locus will accelerate the progress of a second which it complements, but would impede that of another which it duplicates. These patterns of gene frequency change will in turn be reflected in terms of population mean, so that systems of complementary loci can be expected to give rapid but relatively short-lived responses, whereas duplicate loci will tend towards a slower, diminishing, but longer-lasting response.

\section{(b) Equilibria}

For one locus models, maximisation of population mean can occur at intermediate allele frequencies only when there is overdominance $(h>d)$ At this frequency, the first derivative and thus the additive genetic variance are both zero. Selection will tend to reduce the derivative, and so this equilibrium is stable. For a pair of epistatic diploid loci, strict overdominance is no longer necessary as epistatic components are involved. From the model used earlier:

$$
\begin{aligned}
d y / d p=\left(d_{a}+(u-v) i+2 u v j_{a}+(q-p)\left(h_{a}+(u-v) j b+\right.\right. & 2 u v l)) \\
& =E_{a}+(q-p) F_{a}
\end{aligned}
$$

Population mean with respect to this locus (A) is therefore maximised if $p=\left(E_{a}+F_{a}\right) / 2 F_{a}$. Thus $E_{a}$ and $F_{a}$ represent the apparent additive and dominance effects at the $A$ locus, and contain contributions due to epistatic interactions with locus $B$. The optimum frequency, $p^{\prime}$, can therefore only be intermediate when $F_{a}$ exceeds $E_{a}$. The magnitude of this excess itself depends on the allele frequency $(u)$, and is maximum when

when

$$
d\left(F_{a}-E_{a}\right) / d u=2\left(\left(j_{b}-i\right)+(v-u)\left(l-j_{a}\right)\right)=0,
$$

$$
F_{a}-E_{a}=h_{a}-d_{a}+\left(\left(j_{b}-i\right)^{2}+\left(l-j_{a}\right)^{2}\right) / 2\left(l-j_{a}\right) .
$$

An intermediate optimal gene frequency is only possible when this expression is positive, the probability of which is enhanced when the excesses of $j_{b}$ 
over $i$ and $l$ over $j_{a}$ are increased. A similar expression for intermediate optimum allele frequency at $B$ can be easily derived, but a stable equilibrium for the whole system of two loci will depend on simultaneous solutions for $d y / d p=d y / d u=0$.

\section{(ii) Composite populations}

The Taylor expansion has been used to generate a basic model for the representation of the mean value of any randomly mating population in terms of the gene frequencies at loci which are either independent or interacting in pairs. This expansion can thus be used for the comparison of different populations, and can be applied to the problem of the prediction of a composite which is a randomly mating population formed by the pooling of two or more parental populations. For example, if the value of one parent of a two-parent diploid composite is $P 1$, then the value of the second, which differs with respect to gene frequency at a single locus by $x$, is

$$
P 2=P 1+x(d y / d p)+\frac{1}{2} x^{2}\left(d^{2} y / d p^{2}\right),
$$

while the composite is

$$
C=P 1+\frac{1}{2} x(d y / d p)+\frac{1}{8} x^{2}\left(d^{2} y / d p^{2}\right) .
$$

Noting that the hybrid between these parental populations is

then

$$
H=P 1+\frac{1}{2} x(d y / d p),
$$

$$
C=(P 1+P 2) / 4+H / 2,
$$

which is a familiar relation in diploids.

Similar reasoning can be used to show that the heterosis exhibited by a composite over the mean of its $k$ parents with respect to a pair of loci showing only additive, dominance and additive $\times$ additive effects, (i.e. $V_{A D}=V_{D A}=V_{D D}=0$ ) is

$$
-(k-1) / k\left[\frac{1}{2} \sigma_{p}^{2}\left(d^{2} y / d p^{2}\right)+\frac{1}{2} \sigma_{u}^{2}\left(d^{2} y / d u^{2}\right)+\sigma_{u p}\left(d^{2} y / d p . d u\right)\right]
$$

where all three derivatives are constant. The heterosis due to dominance effects thus depends on the variance of gene frequencies among the parents, while the contribution of additive $x$ additive epistasis depends on their covariance. The average heterosis of hybrids (in diploids) is similarly found to be

$$
-\frac{1}{2}\left[\sigma_{p}^{2}\left(d^{2} y / d p^{2}\right)+\sigma_{u}^{2}\left(d^{2} y / d u^{2}\right)+\sigma_{u p}\left(d^{2} y / d p \cdot d u\right)\right]
$$

These expressions can be used to give prediction formulae for diploid composites in terms of the parents and hybrids. In the absence of epistasis,

$$
C_{k}=[(k-1) \bar{H}+P] / k,
$$

which is the usual Sewall-Wright formula (Wright, 1922) where $\bar{P}$ and $\bar{H}$ are the means of the parents and their hybrids respectively. For loci exhibiting only additive and additive $\times$ additive effects,

$$
C_{k}=[2(k-1) \bar{H}-(k-2) \bar{P}] / k .
$$

The above formulae are not applicable to higher levels of ploidy because there is no apparent Taylor expansion for the expectations of hybrids. 
However, this problem can be overcome if composites are used for prediction instead of hybrids. In general, for any level of ploidy showing dominance and/or additive $\times$ additive epistasis:

$$
C_{k}=\left[n(k-m) \bar{C}_{n}-m(k-n) \bar{C}_{m}\right] / k(n-m),
$$

where $k>n>m$, and $\bar{C}_{n}$ and $\bar{C}_{m}$ are the means of all possible $n$ and $m$ parent composites from $k$ parents. In particular, if two parent composites and parents are used $(n=2, m=1)$ the formula recovered is identical to that given by Gallais and Vincourt (1978) for the prediction of diploid synthetics with homozygous parentage. In fact, all the above developments for composite varieties apply to synthetics with homozygous parents, because each of the latter is a special form of random mating population.

No prediction formulae of this type can be derived when higher-order interactions are present, whether the alleles involve one, two, or more loci, as the second-order derivatives are no longer constants.

\section{Disqussion}

The development of the method of formulation of genetic variances for two allele models given here represents a development and simplification of that given by $\mathrm{Li}$ (1957), and allows models with arbitrary levels of ploidy and allelic and non-allelic interaction to be handled easily. The treatment of multiple allelism could probably be catered for by the use of partial derivatives with respect to the frequency at each allele, but this would certainly lead to the loss of the very simplicity which is the chief quality of the method. In any case its main function must be the treatment of models of gene action whose usefulness also depends on the simplicity of biallelism.

The limited application of differential coefficients and associated Taylor series to some aspects of selection and heterosis given here is not intended to be exhaustive, but only to introduce a novel approach to quantitive genetical problems which may turn out to be a useful complement to existing methods.

\section{ReFERENCES}

Arunachalam, v., AND owen, A. R. G. 1971. Polymorphisms with Linked Loci. Chapman and Hall, London.

DALEBroux, M. I962. An extension of the use of orthogonal coefficients in partitioning genetic variances. Heredity, 17, 579-583.

EAston, H. s. 1976. Étude comparative d'effects génetiques chez des plantes diploids at tetraploides isogéniques de Festuca pratensis Huds. Thèse de Doctoral d'État ès Sciences Naturelles, Université de Paris. Sud.

FAlconer, D. S. 1960. Introduction to Quantitative Genetics. Oliver and Boyd, Edinburgh. GALlais, A., AND vincourt, P. 1978. The concept of varietal ability in plant breeding with some results on cocksfoot. Proc. 3rd Mesting Eucarpia Biometrics Section.

KEMPthorne, O. 1957. Introduction to Statistical Genetics. John Wiley, New York.

LI, c. C. 1957. The genetic variance of autotetraploids with two alleles. Genetics, 42, 583-592.

MATHER, K. 1974. Non-allelic interaction in continuous variation of randomly breeding populations. Heredity, 32, 4I4-419.

MAther, K., AND JiNks, J. L. 197I. Biometrical Genetics, 2nd Ed. Chapman and Hall, London.

WRIGHT, s. 1922. The effects of inbreeding and crossbreeding on guinea pigs. U.S. Dept. Agric. Bull., 1121. 